\title{
Radiological Diagnosis and Management of Epistaxis
}

\author{
Antonín Krajina • Viktor Chrobok
}

Received: 24 October 2012/ Accepted: 4 October 2013/Published online: 15 November 2013

(C) The Author(s) 2013. This article is published with open access at Springerlink.com

\begin{abstract}
The majority of episodes of spontaneous posterior epistaxis treated with embolisation are idiopathic in nature. The angiographic findings are typically normal. Specific angiographic signs are rare and may include the following: a tumour blush, telangiectasia, aneurysm, and/or extravasation. Selective internal carotid artery (ICA) angiography may show rare causes of epistaxis, such as traumatic or mycotic aneurysms, which require different treatment approaches. Complete bilateral selective external and internal carotid angiograms are essential to evaluation. The images should be analysed for detection of central retinal blush in the external carotid artery (ECA) and anastomoses between the branches of the ECA and ICA. Monocular blindness and stroke are two of the most severe complications. Embolisation aims to decrease flow to the bleeding nasal mucosa while avoiding necrosis of the nasal skin and palate mucosa. Embolisation is routinely performed with a microcatheter positioned in the internal maxillary artery distal to the origin of the meningeal arteries. A guiding catheter should be placed in the proximal portion of the ECA to avoid vasospasm. Embolisation with microparticles is halted when the peripheral branches of the sphenopalatine artery are occluded. The use of coils
\end{abstract}

\footnotetext{
A. Krajina $(\square)$

Department of Radiology, University Hospital Hradec Kralove and Medical Faculty of Charles University, 50005 Hradec Kralove, Czech Republic

e-mail: antonin.krajina@fnhk.cz

V. Chrobok

Department of Otorhinolaryngology and Head and Neck Surgery, University Hospital Hradec Kralove and Medical Faculty of Charles University, 50005 Hradec Kralove, Czech Republic

e-mail: viktor.chrobok@fnhk.cz
}

is not recommended because recurrent epistaxis may occur due to proximal embolization; moreover, the option of repeat distal embolisation is lost. The success rate of embolisation therapy (accounting for late recurrence of bleeding) varies between 71 and $94 \%$. Results from endoscopic surgery are quite comparable. When epistaxis is refractory to nasal packing or endoscopic surgery, embolisation is the treatment of choice in some centres.

Keywords Posterior epistaxis - Selective percutaneous embolisation - Endovascular intervention

\section{Introduction}

Haemorrhage from the nose is formally referred to as "epistaxis." Haemostasis within the nose can be compromised by mucosal abnormalities, vessel pathology, or coagulation disorders. The aetiology of epistaxis is divided into groups: local and systemic factors. Local factors include the following: trauma, local inflammatory reactions, foreign bodies, postsurgical anatomical deformities, intranasal tumours, chemical inhalants, nasal-prong oxygen administration, and continuous positive airway pressure therapy for obstructive sleep apnoea. Systemic causes of epistaxis include the following: vascular disorders, especially hereditary haemorrhagic telangiectasia (HHT); blood dyscrasias; hematologic malignancies; and drugs affecting the normal clotting mechanism. Epistaxis arising from the anterior septal area (anterior epistaxis) is more common in children and young adults; it is the most common type of epistaxis and is more often venous in origin. This condition is usually responsive to treatment because it is readily accessible for nasal packing if bleeding does not resolve 
spontaneously [1, 2]. Therefore, endovascular therapy is usually not indicated for anterior epistaxis.

Approximately $5 \%$ of epistaxis episodes arise from the posterior and superior portions of the nasal cavity, and the majority of posterior epistaxis episodes arise from the arteries of the septum. Posterior epistaxis is more common in older patients than in children [3] The most common factors associated with posterior epistaxis are hypertension $[4,5]$, acetylsalicylic acid or nonsteroidal anti-inflammatory drug use [6], previous episodes of epistaxis, alcohol use, and anticoagulant use. However, the majority of these cases are idiopathic [7]. Refractory epistaxis is defined as recurrent or persistent bleeding after appropriate packing or multiple episodes of epistaxis during a short period of time, each requiring medical attention. Embolisation for intractable epistaxis was first reported as an alternative to surgery and other methods by Sokoloff [8].

\section{Nonendovascular Therapy for Epistaxis}

The management of epistaxis should begin with general measures, including calming the patient (with sedatives if necessary), application of a cold compress to the nape of the neck, decreasing the blood pressure if the patient is hypertensive, fluid resuscitation, and correction of any underlying coagulopathy. Local measures include the following: various methods of bleeding control, such as local application of haemostatic agents, infiltration of the bleeding area with vasoconstrictive agents, and cautery. Anterior nasal packing is performed by positioning strips of ointment-saturated gauze so as to produce satisfactory pressure on the bleeding mucosa. Posterior nasal packing is an option if anterior packing is insufficient to stop the haemorrhage or if the bleeding originates posteriorly. Posterior nasal packing requires general anaesthesia. A balloon catheter is inflated in the nasopharynx to stop the flow of blood posteriorly, and the anterior part of the nasal cavity is packed. The catheter coming out of the nose is fixed in place [3, 9].The patient should be monitored because posterior nasal packing can lead to nasal trauma, vasovagal reaction, and infection. Traditional gauze packing or balloon devices may be left in the nasal cavity for $\leq 48 \mathrm{~h}$ to prevent such complications. The success rate of posterior packing is reported to be between 48 and $83 \%$. Subsequent therapy can include either surgical endonasal coagulation or ligation [10]. An endoscopic approach has been used for direct electrocauterisation of the active bleeding site and endoscopic ligation of the sphenopalatine and ethmoidal arteries [11]. Surgical intervention is clearly indicated in anterior ethmoid bleeding because the ethmoidal arteries arise from the ophthalmic artery, the embolisation of which is generally considered to be dangerous.

\section{Endovascular Therapy of Epistaxis}

\section{Relevant Anatomy}

Detailed knowledge of the arterial anatomy of this region is important for safe and successful treatment. Stroke and blindness are two of the most severe complications. When retinal opacification from branches of the external carotid artery (ECA) is identified, embolisation must not be performed, and the patient should be referred for surgical treatment [12-15].

The arterial branches involved in epistaxis include the following: the internal maxillary artery (IMA), the facial artery, and the ophthalmic artery (Fig. 1). The other branches of the ECA are rarely involved.

The IMA is divided into three parts based on its relationship to the external pterygoid muscle [16, 17] (Table 1). The proximal portion is referred to as the "mandibular portion" and originates behind the neck of the mandible. The second, or pterygoid, portion is in contact with the external pterygoid muscle and varies in its location in relationship to this muscle. It is most commonly located superficial to the external pterygoid muscle, although in one third of cases, it is deep to this muscle. The middle meningeal artery arises in front of the inferior alveolar artery in the superficial variant and behind the inferior alveolar artery in the deep variant. The third, or pterygopalatine, portion is the terminal portion of the IMA, creating a loop within the pterygopalatine fossa. This vessel is the target for endovascular therapy in the treatment of posterior epistaxis (Table 2) (Fig. 2).

The sphenopalatine artery, which is the branch of the third part of the IMA, leaves the pterygopalatine fossa by way of the sphenopalatine foramen [18] (Fig. 3). This artery is the major arterial supply to the nasal fossa and enters this fossa posteriorly to the superior meatus. Its lateral nasal branches supply the nasal turbinates, and the medial branches supply the nasal septum [19].

The descending palatine artery, another branch of the pterygopalatine portion of the IMA, supplies the nasal fossa. This artery courses through the pterygopalatine canal and emerges from the palatine foramen. It outlines the posterior wall and floor of the maxillary antrum. The terminal branch enters the incisive foramen and supplies the inferior nasal septum. Here, the branch anastomoses with the medial nasal branches of the sphenopalatine artery. The infraorbital artery enters the orbit through the infraorbital fissure and runs through the infraorbital foramen. Its branches supply the cheek, lower eyelid, and upper lip, and nose. This artery does not supply the nasal mucosa.

The ophthalmic artery gives rise to a collateral network with the sphenopalatine artery by way of the anterior and posterior ethmoidal branches. These arteries pass through 
Fig. 1 Schematic arterial supply of the sinonasal cavity. The majority of the posterior epistaxis episodes arise from the septum. The arterial branches involved in epistaxis include the internal maxillary artery, the facial artery, and the ophthalmic artery (Courtesy of V.

Machova)

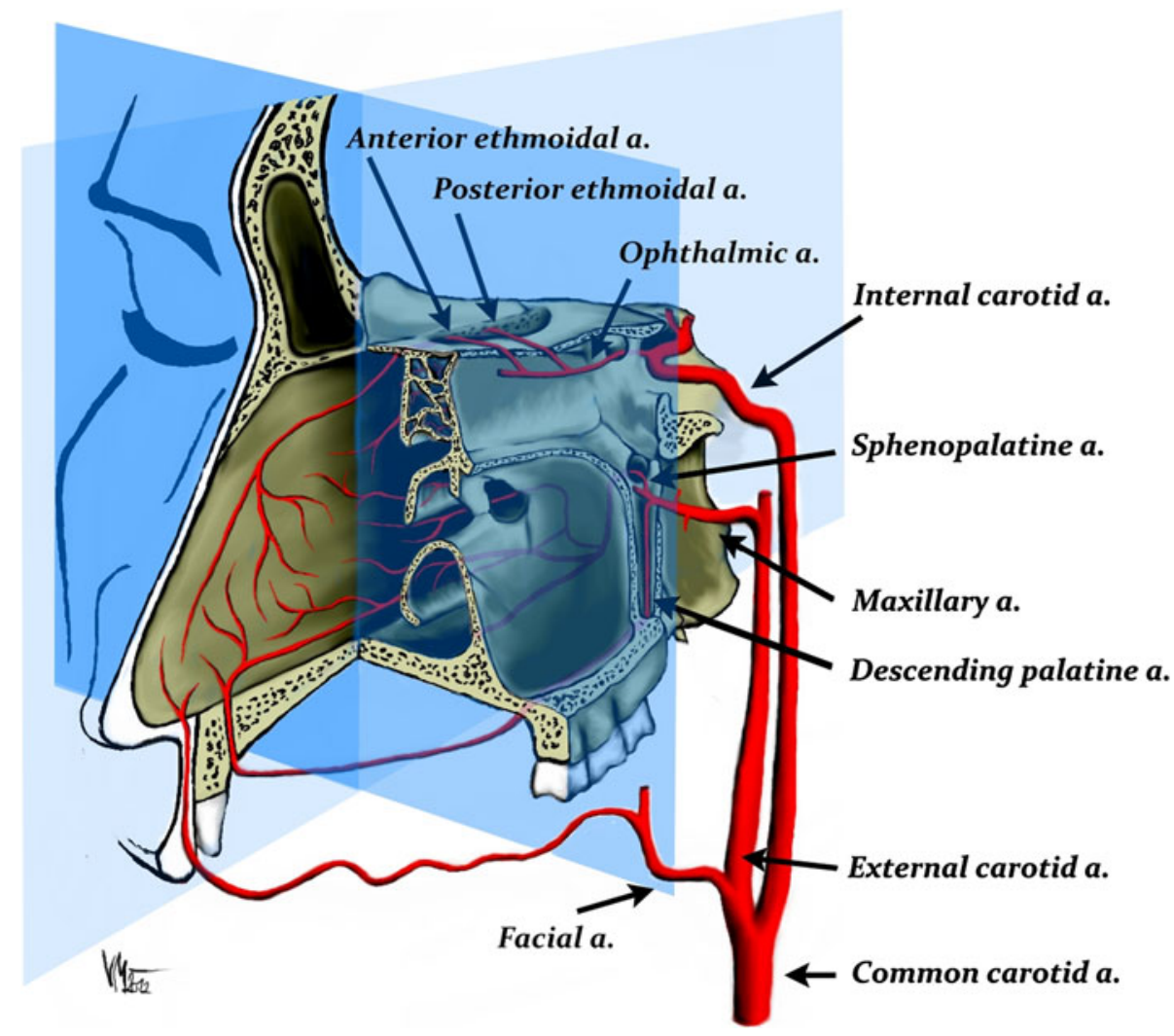

Table 1 Branches of the maxillary artery (modified according Allen et al. [16])
I. First (mandibular) portion
a. Anterior tympanic
b. Deep auricular
c. Middle meningeal ${ }^{\mathrm{a}}$
d. Accessory meningeal ${ }^{\mathrm{a}}$
e. Inferior alveolar
II. Second (pterygoid) portion
a. Deep temporal
b. Pterygoid
c. Masseteric
d. Buccal
III. Third (pterygopalatine) portion
a. Posterior superior alveolar
b. Infraorbital
c. Greater (descending) palatine ${ }^{b}$
d. Artery to foramen rotundum ${ }^{\mathrm{a}}$
e. Artery of pterygoid canal (vidian artery) ${ }^{\mathrm{a}}$
f. Pharyngeal
g. Sphenopalatine ${ }^{b}$

\footnotetext{
${ }^{a}$ Dangerous arteries for embolisation. The pterygoid artery and Vidian artery are considered to be two different branches by Lasjaunias et al. [20]

b These arteries are targets for embolisation
}

Table 2 Distribution of the third (pterygopalatine) portion of the maxillary artery (modified according Allen et al. [16])

\begin{tabular}{lll}
\hline Direction & Branch & Route of exit \\
\hline Lateral & Posterior superior alveolar & Pterygomaxillary fissure \\
Anterior & Infraorbital & Inferior orbital fissure \\
Inferior & Greater palatine & Pterygopalatine canal \\
Medial & Sphenopalatine & Sphenopalatine foramen \\
Posterior & Artery of foramen rotundum & Foramen rotundum \\
& Artery of pterygoid canal & Pterygoid canal \\
& Pharyngeal & Palatinovaginal canal \\
\hline
\end{tabular}

the cribriform plate of the ethmoid. The superior labial artery, a small branch of the facial artery, contributes to the arterial supply of the nasal septum. This branch is not regularly visible on angiographic studies.

The facial artery is in hemodynamic balance with the other branches of the ECA. The preferred arterial supply is established in accordance with local hemodynamic needs [20]. This situation means that the direction of blood flow through anastomoses among the facial, internal maxillary, and ophthalmic arteries depends on the force and rate of injection, proximal vasospasm, and pre-existing occlusion due to ligation or embolisation [15] (Fig. 4). 


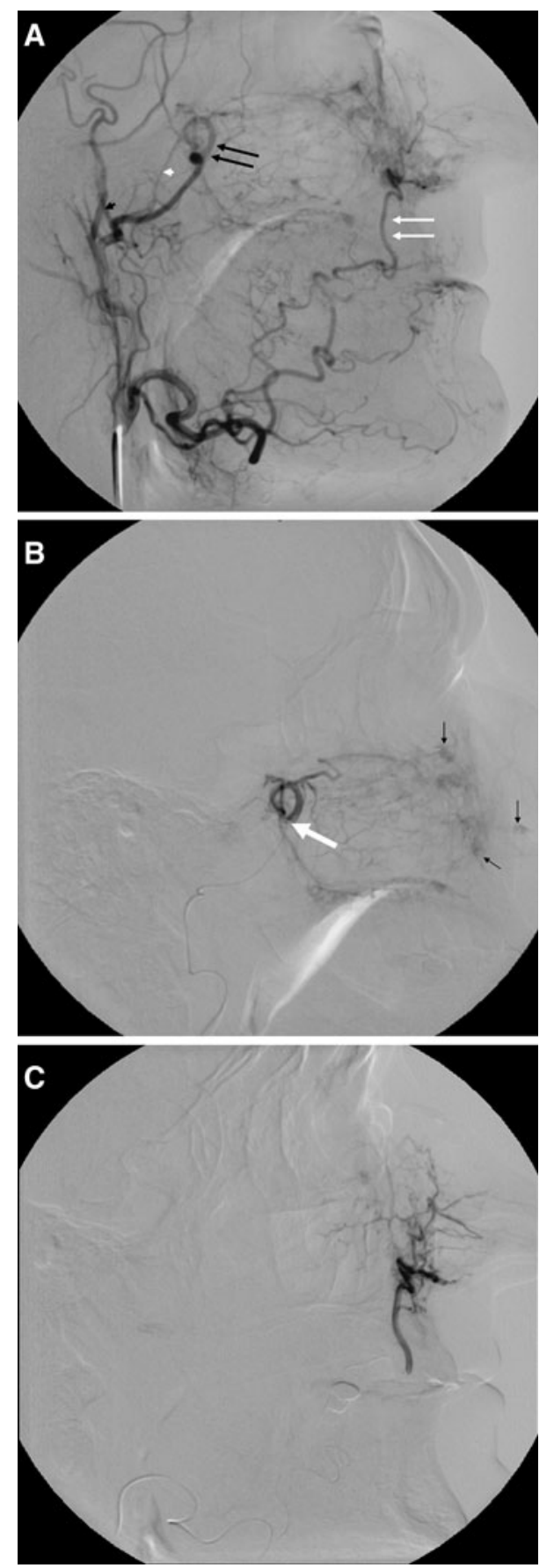

Fig. 2 A External carotid angiogram of a patient with HHT and multiple episodes of severe epistaxis. The target artery for embolisation is the sphenopalatine artery (double black arrows) and the terminal portion of the facial artery (double white arrows). A microcatheter for embolisation should be placed distal to the middle meningeal artery (small black arrowhead) and accessory meningeal artery (small white arrowhead). B Selective internal maxillary angiogram showing the position of a microcatheter (large white arrow). There are separate mucosal hypervascular areas caused by telangiectasias (small black arrows). C Selective facial angiogram that shows the supply to the nasal cavity
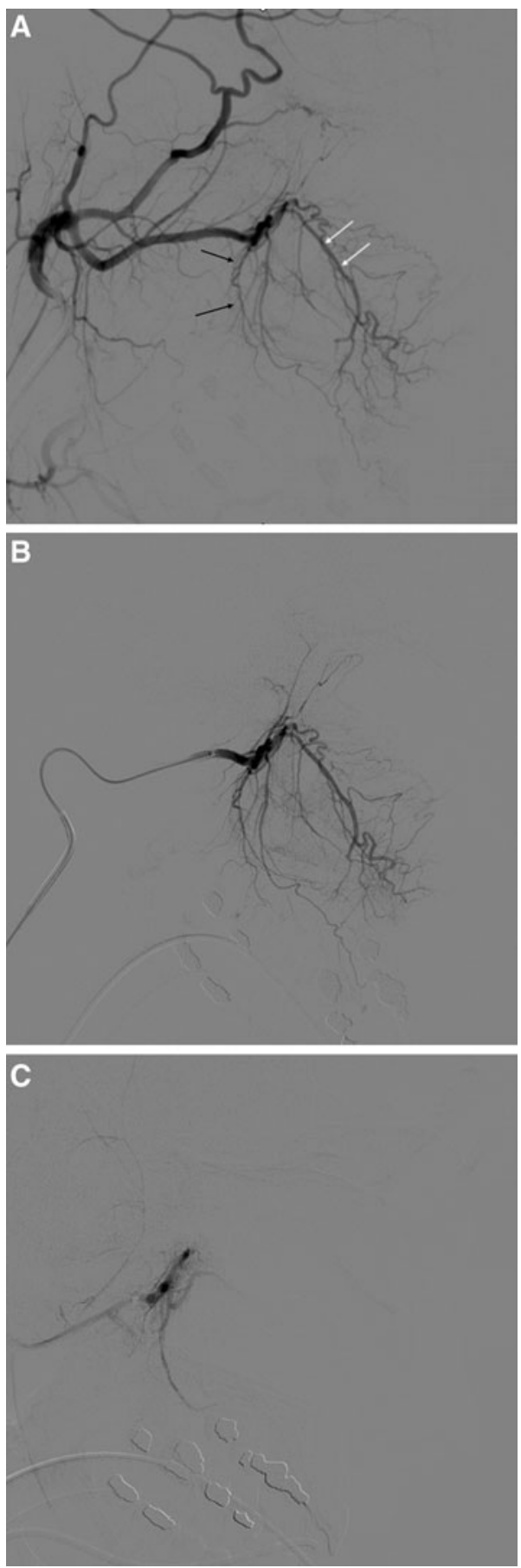

Fig. 3 A Angiographic anatomy of the distal internal maxillary artery. The descending palatine artery (black arrows) outlines the posterior wall and floor of the maxillary antrum. The infraorbital artery (double white arrows) enters the orbit through the infraorbital fissure. B Selective distal internal maxillary artery angiogram in a lateral view. C Completion angiogram after embolisation with microparticles (Courtesy of J. J. Vitek) 

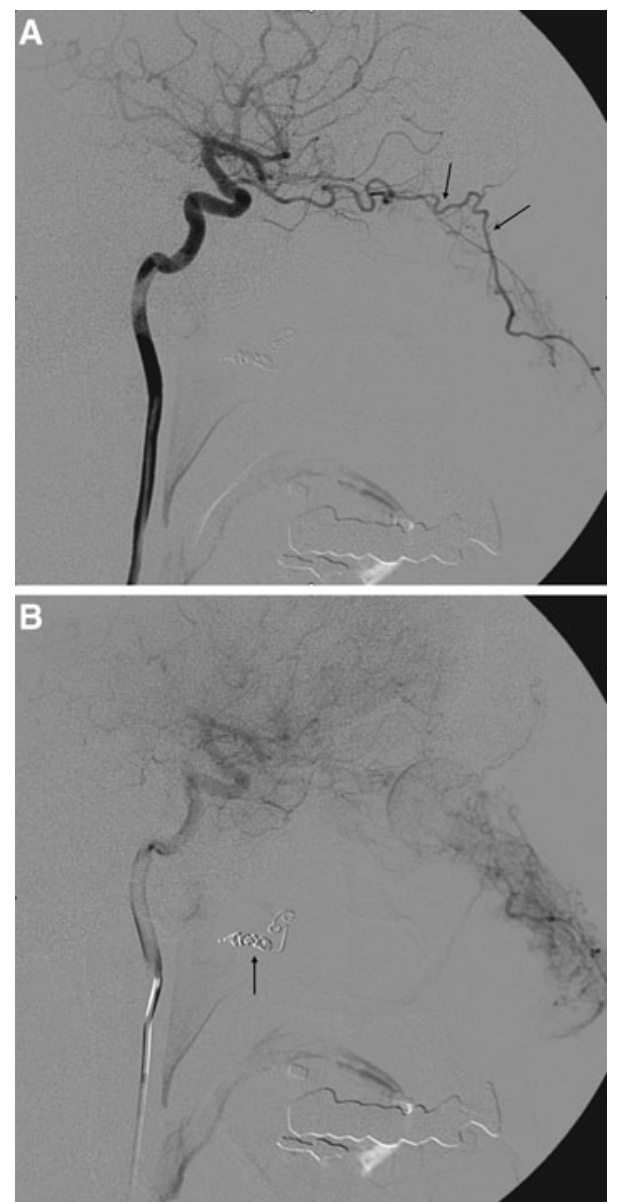

Fig. 4 A Internal carotid angiogram in a patient with recurrent epistaxis. There is a rich collateral supply to the nasal cavity from the ophthalmic artery (arrows). B The cause of such a collateral pathway is previous proximal embolisation of the internal maxillary artery using coils (arrow) (Courtesy of Dr. M. Vavrova)

Some investigators state that a venous plexus, called Woodruff's plexus, is a common source of bleeding in cases of posterior epistaxis. This plexus is located in the posterior part of the inferior meatus and around the choanae [21].

\section{Endovascular Therapy for Idiopathic Posterior Epistaxis}

\section{Preprocedure Preparation}

Computed tomography (CT) or magnetic resonance imaging is not typically indicated for the investigation of epistaxis unless tumour or other local diseases are suspected as an underlying cause. Idiopathic epistaxis is classified after exclusion of local or systemic causes. The interventional radiologist should be aware of the laterality of the nose bleed, the current severity of bleeding, and the patient's history. These factors are crucial in deciding whether general anaesthesia is required for the procedure. General anaesthesia is routinely used only in children, noncompliant patients, and patients having uncontrolled epistaxis with possible compromise of the airway. The majority of embolisation procedures can be performed with intravenous sedation and analgesia with standard monitoring of blood pressure, electrocardiogram, and pulse oxymetry. Nasal packing, which is radiopaque, should be avoided in patients who might be candidates for embolotherapy.

\section{Underlying Coagulopathy}

The presence of any underlying coagulopathy should be evaluated because embolisation with polyvinylalcohol (PVA) microparticles is more effective in patients with normal platelet and plasma protein functions [22].

A proposed protocol for anticoagulants and epistaxis is as follows [1,23]:

1. Obtain full blood count, platelet count, and international normalised ratio (INR)

2. Patients with metallic heart valves on warfarin should be kept only on a therapeutic level of INR. A cardiologist should be contacted if life-threatening bleeding occurs

3. Patients with coronary stents treated with acetylsalicylic acid or other antiplatelet therapy should be discussed with a cardiologist for possible platelet transfusion in cases of life-threatening epistaxis

\section{Angiography and Embolisation}

Complete selective external and internal carotid angiograms are essential to evaluation. Angiograms should be analysed for the detection for arterial variants and anastomoses between branches originating from the external carotid and internal carotid arteries [14, 15]. Standard catheterisation of the common carotid artery (CCA) is performed. Depending on the type of the aortic arch, various diagnostic and guiding catheters are selected. The most frequently used are vertebral, Berenstein, Davis, Simmons, or Vitek catheters. A $5 \mathrm{~F}$ or $6 \mathrm{~F}$ guide catheter permits angiography while a microcatheter is in the target artery. A standard continual pressure flush with heparinised normal saline must be used to prevent the backflow of blood into the guiding catheter.

The first injection of $7-10 \mathrm{ml}$ of iodinated contrast medium into the CCA is performed using the posterioanterior and lateral views. The angiogram should show any potential arterial pathology at the common carotid artery bifurcation, including atherosclerotic plaque or occlusion of the internal carotid artery (ICA) (Table 3). Because the 
Table 3 Angiographic and embolisation protocol in epistaxis
A. The ICA angiogram
1. Start on the side of epistaxis (if identified)
2. Check the carotid bifurcation for stenosis or occlusion
3. Look for central retinae blush, ophthalmic retina feeders to the nasal mucosa
4. Exclude source of epistaxis from the ICA
B. The ECA angiogram
1. Position the guide catheter with its tip just within the ECA to avoid vasospasm
2. Exclude filling of the ophthalmic artery (retinae blush on lateral view)
3. Exclude collaterals to the ICA
4. Show any arterial pathology as a source of bleeding
C. Embolisation of the IMA

1. Place microcatheter into the pterygopalatine portion of the IMA sufficiently distal to the accessory or middle meningeal arteries, preferably distal to the infraorbital artery

2. Perform flow-directed embolisation with PVA particles $(250-500 \mu \mathrm{m})$ in nondiluted contrast agent using a 1-ml syringe

3. Avoid reflux and coil embolisation

D. Perform contralateral ICA and ECA angiograms as well as IMA embolisation

E. Remove the nasal packing and check the nasal pathway for bleeding; if no bleeding appears for $15 \mathrm{~min}$, remove the microcatheter and perform a completion ECA angiogram

F. Perform embolisation of the ipsilateral facial artery with the microcatheter placed as distally as possible (at least distal to the submandibular part of the facial artery) using 250- to $350-\mu \mathrm{m}$ PVA particles if significant contribution to the nasal mucosa is angiographically visible

majority of posterior epistaxis episodes treated by embolisation are idiopathic, angiographic findings in these cases may be normal. Specific angiographic signs are rare and may include the following: tumour blush, telangiectasia, traumatic pseudoaneurysm, and even contrast extravasation. Selective ICA angiography may show other sources of epistaxis, such as mycotic or traumatic aneurysm $[12,13]$.

Arterial vasospasm is an undesired but common consequence of forceful manipulation, particularly manipulation with the tip of the guiding catheter in the proximal part of the ECA or microwire manipulation in the branches of the ECA. The presence of proximal vasospasm impairing the arterial flow in the access artery can alter the hemodynamic pattern of the arterial feeders in the target area, thus rendering embolisation impossible. Vasodilators may be administered intra-arterially to treat or prevent vasospasm [24, 25].

A microcatheter that will allow the use of microparticles $\leq 500 \mu \mathrm{m}$ in diameter is placed coaxially in the pterygopalatine segment of the IMA, and angiography is performed to determine if there are any large anastomoses involving the ICA or ophthalmic artery. The most useful projection to evaluate the ECA, IMA, and retinal blush is

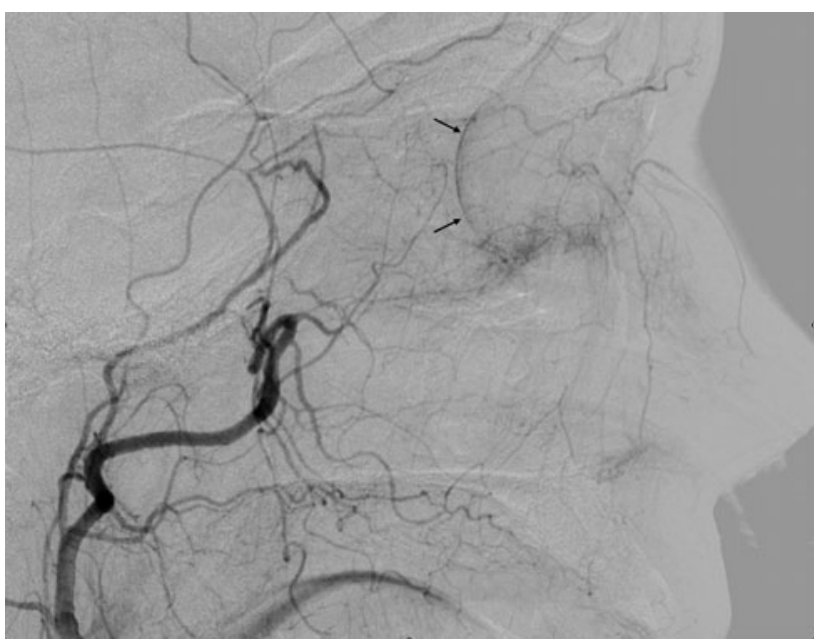

Fig. 5 External carotid angiogram showing the supply of the retina (arrows)

the lateral view (Figs. 4B, 5). Most commonly, supply of the ophthalmic artery from the ECA branches occurs due to collateralisation observed in chronic ICA stenosis or occlusion. The terminal branches of the pterygopalatine segment are best seen in frontal views.

Microparticle embolisation is performed using a standard free-flow technique. A wedged position of the microcatheter should be avoided (Fig. 3B). Ideally, the capillary bed of the nasal cavity is slowly saturated with embolic microparticles. This goal is accomplished using fluoroscopic guidance in the lateral view to assess blood flow velocity in the artery, potential backflow, and reflux of the embolic agent (Fig. 3C). During embolisation, the operator should perform selective angiograms, the quality of which enables not only assessment of occlusion progress but also the potential filling of collaterals. The tip of a microcatheter is placed into the IMA distal to the origin of the middle meningeal and accessory meningeal artery due to the anastomoses with the ICA (Fig. 2A) In addition to the medial and lateral branches of the sphenopalatine artery, it is also desirable to embolise the descending palatine artery (Fig. 3A). This artery originates distal to the infraorbital artery (Fig. 3A). Reflux of embolic particles into the infraorbital artery is generally considered to be safe. The penetration of microparticles into the middle deep temporal artery may cause pain and trismus after embolisation. The embolic microparticles are mixed with nondiluted contrast medium to achieve the best visibility during injection using a 1-ml syringe. The mixture should not be too concentrated and must be continually mixed because the PVA microparticles have a tendency to sediment and occlude the microcatheter. Spherical microparticles make a more stable mixture. Spherical microparticles can penetrate deeper into arteries than PVA microparticles of the same size. Particles should lodge distal enough to control haemorrhage and yet proximal enough to 
preserve the distal supply of the terminal alar artery, which supplies the skin of the nose. The PVA microparticles $200-500 \mu \mathrm{m}$ in size are usually selected. It is useful to talk to the patient if he or she is awake to show any ischaemic neurological deficit as soon as possible. In the sleeping patient, we must rely on anatomical angiographic studies and a thorough knowledge of the anatomy [26].

Embolisation is halted when the peripheral branches of the sphenopalatine segment are not opacified and the arterial tree seems to be debranched (Fig. 3C). The amount of microparticles required varies. Special care must be taken as soon as the flow begins to slow because even small additional amounts of microparticles may result in unexpected arterial stasis or even reflux.

The strategy for embolisation in patients with idiopathic epistaxis relies on the identification of the side of bleeding and the collateral pathways involving the contralateral IMA and the ipsilateral facial artery (Fig. 2C). The distribution of embolised arteries varies significantly in published series. The bilateral IMAs together with the ipsilateral facial artery were embolised in 27-48 \%, the bilateral IMAs in 15-35\%, and the unilateral IMA in $13-70 \%$ of cases. The bilateral IMAs are embolised if the side of haemorrhage is unknown or bilateral IMAs and ipsilateral facial artery if the side of haemorrhage is known. There also exist published case reports describing embolisation of the ascending pharyngeal and accessory meningeal supply to the nasal fossa for the management of epistaxis [27-29].

\section{Nasal Packing Removal}

Two different strategies regarding nasal packing removal have been reported in the literature. Some operators remove the packs while the catheters are still in the arteries, check the nasal pathway for bleeding, and finish the procedure if the nasal pathway remains dry for $15 \mathrm{~min}$. Persistent bleeding influences the decision to embolise the contralateral IMA or the ipsilateral facial artery [30]. Other practitioners leave the packs in place to facilitate the synergistic effects of external compression and devascularisation. Nasal packing removal is influenced by the duration of its placement, the severity and intractability of the epistaxis, and occasionally by the time of day the procedure is performed. Care must be taken to prevent the aspiration of pooled blood into the respiratory tract.

\section{Clinical Success}

The primary success rates of epistaxis embolization therapy range from 71 to $95 \%$ in the literature (Table 4 ). The success rate is influenced by the embolisation protocol used. Embolisation of the bilateral distal IMAs and ipsilateral distal facial artery with $200-\mu \mathrm{m}$ PVA microparticles significantly decreases short-term failure rates and carries a very low risk for ischemic complications. The rate of early recurrence decreases with embolisation of more arteries [22]. Patients taking oral anticoagulants or antiplatelet medication are noted to have greater rate of early rebleeding than patients not taking these medications [22].

Failures of embolisation therapy to control epistaxis are due to dominant ICA supply by way of the anterior and/or posterior ethmoid arteries. This arterial supply requires external surgical ligation. Late rebleeds are dependent on the underlying pathology and are more frequent with HHT. Often these patients are included in studies involving idiopathic epistaxis [30].

\section{Surgical Versus Endovascular Therapy}

The decision between surgery and embolisation should be based on the individual patient's comorbidities, the anatomical setting, and the availability of adequate interventional radiology [11]. There are retrospective studies available comparing IMA ligation (with or without ethmoid artery ligation) [11] with PVA embolisation of the bilateral IMAs and ipsilateral facial artery [31] for the treatment of refractory epistaxis. The embolised arteries were not specified in one of those studies [11]. The success rates for IMA ligation and embolisation were 89 and $94 \%$, respectively [31]. The other study reported success rates of 73 and $79 \%$, respectively [11]. The investigators in both studies concluded that complication and failure rates of IMA ligation and embolisation were comparable. However, although the major complication rates were not significantly different between the two therapies, the complications associated with embolisation were more serious than those associated with IMA ligation.

The advantages of PVA embolisation include the use of local anaesthesia; pretreatment angiogram, which may show less common causes of epistaxis; distal embolisation; and shorter hospitalisation times. Embolisation is indicated in patients with cardiovascular instability. However, embolisation is available only in specialised centres. Furthermore, if bleeding originates from the ethmoid arteries, embolisation by way of the ophthalmic artery is considered to be hazardous. Ophthalmic artery embolisation should be avoided, even in cases of long-standing blindness in the ipsilateral eye, because retinal necrosis may lead to autoimmune retinal inflammation and loss of vision in the contralateral eye [15]. In the presence of dangerous collaterals, the embolisation material must be exchanged for microcoils, which results in a more proximal and less effective embolisation or referral for surgical treatment. Surgical ligation of the IMA with or without anterior ethmoid artery ligation seems to be the more common technique and requires general anaesthesia in all cases. 
Table 4 Review of studies on posterior epistaxis treated with embolisation

\begin{tabular}{|c|c|c|c|c|c|c|c|}
\hline $\begin{array}{l}\text { First author } \\
\text { (reference) }\end{array}$ & Year $^{\mathrm{a}}$ & $\begin{array}{l}\text { No. of } \\
\text { patients }\end{array}$ & $\begin{array}{l}\text { Mean age } \\
\text { (year) }\end{array}$ & $\begin{array}{l}\text { No. of } \\
\text { procedures }\end{array}$ & $\begin{array}{l}\text { Embolic material } \\
\text { for ECA branches }\end{array}$ & $\begin{array}{l}\text { Immediate clinical } \\
\text { success }(\%)^{\mathrm{b}}\end{array}$ & $\begin{array}{l}\text { Severe } \\
\text { complications }(\%)^{\mathrm{c}}\end{array}$ \\
\hline Vítek [30] & 1991 & 30 & 62 & 34 & GS & 87 & 3.3 hemiparesis \\
\hline Elden [32] & 1994 & 97 & 53 & 108 & PVA & 88 & 2 stroke, skin slough \\
\hline Elahi [57] & 1995 & 57 & 53.1 & 54 & PVA & 91 & 6 stroke \\
\hline Tseng [7] & 1998 & 112 & 55 & 114 & PVA, GS & 91 & 0.9 stroke \\
\hline Moreau [58] & 1998 & 45 & 48.8 & 46 & PVA & 95 & 4 \\
\hline Leppanen [59] & 1999 & 37 & 53 & 38 & PVA, coils & 89 & 0 \\
\hline Oguni [60] & 2000 & 37 & 57.3 & 40 & GS & 95 & 0 \\
\hline Andersen [33] & 2005 & 22 & 59 & 30 & PVA, spheres & 87 & 5 nose necrosis \\
\hline Christensen [61] & 2005 & 70 & 59.1 & 70 & PVA, GS, coils & 87 & 1 stroke \\
\hline Sadri [34] & 2006 & 14 & 57 & 15 & PVA & 71 & 7 nose necrosis, palate necrosis \\
\hline Fukutsuji [62] & 2008 & 22 & 56.8 & 23 & GS, coils & 77.3 & 0 \\
\hline Strach [35] & 2011 & 48 & 58.7 & 53 & PVA, coils & 93.5 & 4 nose necrosis, hemiparesis \\
\hline Gottumukkala [56] & 2013 & 84 & 63.8 & 85 & PVA & 89 & 1 \\
\hline
\end{tabular}

$G S=$ gelatin sponge

a The table includes studies with selective embolisation using microcatheters

b The overall clinical success also depended on the proportions of patients with HHT

${ }^{c}$ Definitions of severe stroke were variable

\section{Complications}

Embolisation should decrease flow to the bleeding nasal mucosa while avoiding necrosis of the nasal skin or palate mucosa. Skin necrosis requires plastic surgical repair [3235]. Smaller particles $(45-150 \mu \mathrm{m})$ carry a greater risk of collateral tissue ischemia, including ischemia of the vasa nervorum [36].

The most frequent causes of neurologic deficit and blindness [37] after embolisation in the ECA territory are reflux of emboli due to vasospasm, nonselective injection, injection of excessive particles at an excessive rate, and failure to recognise dangerous anastomoses due to incomplete angiograms and inadequate analysis [27, 38].

Minor complications include postembolisation ischemic syndrome, which typically lasts for a short time and requires only symptomatic therapy. Acute ischemic sialadenitis has been reported after reflux of microparticles into the proximal branches of the facial artery [39].

\section{Management of Angiographically Proven Causes of Epistaxis}

\section{Traumatic Epistaxis}

The clinical presentation of traumatic epistaxis is lifethreatening haemorrhage at the time of trauma [40]. CT imaging is indicated in all cases of suspected arterial epistaxis. Angiogram may show focal active extravasation, pseudoaneurysm [41], or arterial pseudo-occlusion. The embolisation technique for these cases differs from that for idiopathic epistaxis. The tip of the microcatheter must be as close as possible to the arterial pathology. In the case of possibly dangerous collaterals, microcoils are preferred. The most common sources of bleeding are the sphenopalatine and facial arteries. In a significant number of cases, bilateral extravasation is present [42].

When trauma or infection [43] of the ICA arterial wall results in pseudoaneurysm formation, the patient can present with delayed epistaxis. This pseudoaneurysm should be expected in the cervical, petrous, or cavernous portions of the ICA [44]. Arterial injuries during craniofacial surgery are uncommon but have been well described [45-48].

\section{Juvenile Nasopharyngeal Angiofibroma and Other} Tumours

Juvenile nasopharyngeal angiofibroma (JNA) is a benign hypervascular tumour that occurs in young males between 8 and 23 years of age. Symptoms are related to the size and direction of spread. These symptoms may include recurrent epistaxis (observed in $59 \%$ of cases), which may be lifethreatening. The lesion originates from the posterolateral wall of the nasal cavity in close proximity to the superior aspect of the middle sphenopalatine foramen, the attachment site of the posterior part of the middle turbinate.

The systematic angiographic protocol for JNA begins with injection in the contralateral CCA followed by injections in the ICA ipsilateral common and internal 
carotid arteries. Next, ipsilateral external carotid angiography for evaluation of the maxillary and ascending pharyngeal arteries is performed. The maxillary, accessory meningeal, ascending pharyngeal, and ascending palatine arteries are most commonly embolised vessels. If the tumour crosses the midline, additional embolisation of the appropriate contralateral feeders is performed.

In cases with extensive tumour spread, the supply from the ICA branches may be embolised if possible, especially if the ICA must be sacrificed before surgery. Neurologic deficit is the most feared complication of preoperative embolisation. Blindness, in particular, is of major concern as a result of involvement of the ethmoidal blood supply [38].

Since the introduction of embolisation of JNA by Roberson [49], several studies have been published proving the benefit of preoperative embolisation by decreasing blood loss and operative times. Other sinonasal neoplasms that can cause life-threatening epistaxis include nasopharyngeal carcinoma and hemangiopericytoma [13].

\section{Epistaxis in HHT}

HHT, a hereditary disease, is associated with skin and mucosal telangiectasias and arteriovenous shunts most commonly involving the lung, brain, and liver. It is easily recognised in individuals displaying the classic triad of epistaxis, telangiectasia, and a relevant family history [50].

Epistaxis is caused by spontaneous bleeding from telangiectasias of the nasal mucosa (Fig. 2B). Recurrent epistaxis begins by the age of $10-21$ years and the severity of the episodes increases with age [51].

Among numerous local and surgical treatments, transcatheter embolotherapy has been used with the intent of decreasing the number and severity of epistaxis episodes. Microparticle embolisation should decrease flow to the nasal mucosa, but care must be taken to leave the proximal arteries patent. Embolisation with coils should be avoided because the artery cannot be made again accessible distal to the proximal occlusion in recurrent epistaxis. Preventive embolisation is considered to have a questionable longterm effect [32, 52].

\section{Conclusion}

Ninety five per cent of all epistaxis episodes have their origin in the anterior septum and are easily controlled with local endonasal therapy using anterior packing if bleeding does not resolve spontaneously. Posterior epistaxis represents $5 \%$ of cases and is more difficult to treat. The placement of posterior nasal packs may predispose patients toward vasovagal reaction, infection, and significant discomfort [53-56].
Endoscopic ligation or electrocauterisation of the sphenopalatine and anterior ethmoidal arteries is a routine technique for local control of active bleeding. Embolisation of the distal branches of the bilateral IMAs and ipsilateral distal branches of the facial artery is the preferred therapy in patients with poor cardiovascular status because this procedure can be performed with the patient under local anaesthesia in a majority of cases [22]. Embolisation is also used in the treatment of epistaxis refractory to previous endonasal or surgical therapy, including IMA ligation. Rare but major complications of embolisation therapy are mainly due to the penetration of embolic material into the intracranial and ophthalmic arteries. Embolisation therapy is relatively contraindicated when the bleeding originates mainly from the ethmoid branches of the ophthalmic artery or in the presence of anastomoses between the ECA and the ICA. In our experience, close collaboration between the otorhinolaryngologist and the interventional radiologist provides the best chance of controlling posterior epistaxis.

Conflict of interest Antonín Krajina and Viktor Chrobok have no conflicts of interest.

Open Access This article is distributed under the terms of the Creative Commons Attribution License which permits any use, distribution, and reproduction in any medium, provided the original author(s) and the source are credited.

\section{References}

1. Melia L, McGarry GW (2011) Epistaxis: update on management. Curr Opin Otolaryngol Head Neck Surg 19:30-35

2. Shukla PA, Chan N, Duffis EJ, Eloy JA, Prestigiacomo CJ, Gandhi CD (2012) Current treatment strategies for epistaxis: a multidisciplinary approach. J Neurointerv Surg 5(2):1-6

3. Chiu TW, McGarry GW (2007) Prospective clinical study of bleeding sites in idiopathic adult posterior epistaxis. Otolaryngol Head Neck Surg 137:390-393

4. Schlosser RJ (2009) Epistaxis. N Engl J Med 360:784-789

5. Knopfholz J, Lima-Junior E, Précoma-Neto D, Faria-Neto JR (2009) Association between epistaxis and hypertension: a 1 year follow-up after an index episode of nose bleeding in hypertensive patients. Int J Cardiol 134:e107-e109

6. Soyka MB, Rufibach K, Huber A, Holzmann D (2010) Is severe epistaxis associated with acetylsalicylic acid intake? Laryngoscope 120:200-207

7. Tseng EY, Narducci CA, Willing SJ, Sillers MJ (1998) Angiographic embolisation for epistaxis: a review of 114 cases. Laryngoscope 108:615-619

8. Sokoloff J, Wickbom I, McDonald et al (1974) Therapeutic percutaneous embolisation in intractable epistaxis. Radiology 111:285-287

9. Monte EC, Belmont MJ, Wax MK (1999) Management paradigms for posterior epistaxis: a comparison of costs and complications. Otolaryngol Head Neck Surg 121(1):103-106

10. Klotz DA, Winkle MR, Richmon J, Hengerer AS (2002) Surgical management of posterior epistaxis: a changing paradigm. Laryngoscope 112:1577-1582 
11. Cullen MM, Tami TA (1998) Comparison of internal maxillary artery ligation versus embolisation for refractory posterior epistaxis. Otolaryngol Head Neck Surg 118:636-642

12. Koh E, Frazzini VI, Kagetsu NJ (2000) Epistaxis: vascular anatomy, origins, and endovascular treatment. Am J Roentgenol 174:845-851

13. Willems PWA, Farb RI, Agid R (2009) Endovascular treatment of epistaxis. Am J Neuroradiol 30:1637-1645

14. Lasjaunias P, Marsot-Dupuch K, Doyon D (1979) The radioanatomical basis of arterial embolisation for epistaxis. J Neuroradiol 6:45-53

15. Russell EJ (1986) Functional angiography of the head and neck. Am J Neuroradiol 7:927-936

16. Allen WE III, Kier EL, Rothman SLG (1973) The maxillary artery: normal arteriographic anatomy. Am J Roentgenol 118(3):517-527

17. Osborn AG (1978) The nasal arteries. Am J Roentgenol 130:89-97

18. Hadoura L, Douglas C, McGarry GW, Young D (2009) Mapping surgical coordinates of the sphenopalatine foramen: surgical navigation study. J Laryngol Otol 123:742-745

19. Chiu T (2009) A study of the maxillary and sphenopalatine arteries in the pterygopalatine fossa and at the sphenopalatine foramen. Rhinology 47:264-270

20. Lasjaunias P, Berenstein A, TerBrugge KG (2001) Anatomy and variations of the extracranial carotid artery branches. In: Lasjaunias P, Berenstein A, TerBrugge KG (eds) Surgical neuroangiography, vol 1., Clinical vascular anatomy and variation (2nd ed)Springer, Berlin, pp 292-385

21. Chiu TW, Shaw-Dunn J, McGarry GW (2008) Woodruff's plexus. J Laryngol Otol 122:1074-1077

22. Gottumukkala R, Kadkhodayan Y, Moran CHJ, Cross DWT III, Derdeyn CP (2013) Impact of vessel choice on outcomes of polyvinyl alcohol embolisation for intractable idiopathic epistaxis. J Vasc Interv Radiol 24:234-239

23. Walker FDL, Rutter C, McGarry GW (2008) The use of anticoagulants in epistaxis patients. Rhinology 46:346

24. Kurata A, Miyasaka Y, Tanaka C et al (1996) Prevention of complications during endovascular surgery on the external carotid arteries, with special reference to use of nitropaste and the Lidocaine test. Interv Neuroradiol 2:193-200

25. Smith TP (2006) Embolisation in the external carotid artery. J Vasc Interv Radiol 17:1897-1913

26. Krajina A, Cesak T, Zelenak K, Rehak S (2011) Therapeutic embolisation of cranial tumours. In: Abujamra AL (ed) Diagnostic techniques and surgical management of brain tumours. Intech, Rijeka, pp 493-516

27. Hacein-Bey L, Rosenbloom JS, Pile-Spellman J (1997) Anastomoses in recurrent epistaxis. J Vasc Interv Radiol 8:535-538

28. Duncan IC, Dos Santos C (2003) Accessory meningeal arterial supply to the posterior nasal cavity: another reason for failed endovascular treatment of epistaxis. Cardiovasc Interv Radiol 26:488-491

29. Breda SD, Choi IS, Persky MS, Weiss M (1989) Embolisation in the treatment of epistaxis after failure of internal maxillary artery ligation. Laryngoscope 99:809-813

30. Vítek JJ (1991) Idiopathic intractable epistaxis: endovascular therapy. Radiology 181:113-116

31. Strong EB, Bell A, Johnson LP, Jacobs JM (1995) Intractable epistaxis: transantral ligation versus embolisation: efficacy review and cost analysis. Surgery 113:674-678

32. Elden L, Montanera W, TerBrugge K et al (1994) Angiographic embolisation for the treatment of epistaxis: a review of 108 cases. Otolaryngol Head Neck Surg 111:44-50

33. Andersen PJ, Kjeldsen AD, Nepper-Rasmussen J (2005) Selective embolisation in the treatment of intractable epistaxis. Acta Otolaryngol 125:293-297
34. Sadri M, Midwinter K, Ahmed A, Parker A (2006) Assessment of safety and efficacy of arterial embolisation in the management of intractable epistaxis. Eur Arch Otorhinolaryngol 263:560-566

35. Strach K, Schröck A, Wilhelm K et al (2011) Endovascular treatment of epistaxis: indications, management, and outcome. Cardiovasc Interv Radiol 34:1190-1198

36. Geibprasert S, Pongpech S, Armstrong D, Krings T (2009) Dangerous extracranial-intracranial anastomoses and supply to the cranial nerves: vessels the neurointerventionalist needs to know. Am J Neuroradiol 30:1459-1468

37. Ashwin PT, Mirza S, Ajithkumar N, Tsaloumas MD (2007) Iatrogenic central retinal artery occlusion during treatment for epistaxis. Br J Ophthalmol 91(1):122-123

38. Lasjaunias P (1980) Nasopharyngeal angiofibromas: hazards of embolisation. Radiology 136:119-123

39. Duncan IC, Spiro FI, van Staden D (2004) Acute ischemic sialadenitis following facial artery embolization. Cardiovasc Interv Radiol 27(3):300-302

40. Bynoe RP, Kerwin AJ, Parker HH III et al (2003) Maxillofacial injuries and life-threatening haemorrhage: treatment with transcatheter arterial embolisation. J Trauma 55:74-79

41. Kurata A, Kitahara T, Miyasaka Y, Ohwada T, Yada, Kan S (1993) Superselective embolisation for severe traumatic epistaxis caused by fracture of the skull base. Am J Neuroradiol 14:343-345

42. Komiyama M, Misao N, Masanori K, Tatsuhiro S, Arito K (1998) Endovascular treatment of intractable oronasal bleeding associated with severe craniofacial injury. J Trauma 44:330-334

43. Hurst RW, Judkins A, Boler W, Chu A, Loevner LA (2001) Mycotic aneurysm and cerebral infarction resulting from fungal sinusitis: imaging and pathologic correlation. Am J Neuroradiol 22(5):858-863

44. Chen D, Concus AP, Halbach VV, Cheung SW (1998) Epistaxis originating from traumatic pseudoaneurysm of the internal carotid artery. Diagn Endovasc Ther 108:326-331

45. Raymond J, Hardy J, Czepko R, Roy D (1997) Arterial injuries in transsphenoidal surgery for pituitary adenoma: the role of angiography and endovascular treatment. Am J Neuroradiol 18:655-665

46. Duncan IC, Fourie PA (2004) Acute severe epistaxis due to iatrogenic maxillary pseudoaneurysm: emergency control of haemorrhage by carotid compression and early recurrence after coil embolisation. Interv Neuroradiol 10:269-272

47. Schmerber S, Vasdev A, Chahine K, Tournaire R, Bing F (2008) Internal carotid false aneurysm after thermocoagulation of the Gasserian ganglion. Otol Neurotol 29:673-675

48. Schröck A, Jako M, Strach K et al (2012) Transarterial endovascular treatment in the management of life-threatening intraand postoperative haemorrhages after otorhinolaryngological surgery. Eur Arch Otorhinolaryngol 269:1677-1683

49. Roberson GH, Biller H, Sessions D et al (1972) Presurgical internal maxillary artery embolisation in juvenile angiofibroma. Laryngoscope 82:1524-1532

50. Shovlin CL, Guttmacher AE, Buscarini E et al (2000) Diagnostic criteria for hereditary hemorrhagic telangiectasia (Rendu-OslerWeber syndrome). Am J Med Genet 91:66-67

51. Guttmacher AE, Marchuk DA, White RI Jr (1995) Hereditary hemorrhagic telangiectasia. N Engl J Med 333(14):918-924

52. Weaver EM, Chaloupka JC, Putman C et al (1999) Effect of internal maxillary arterial occlusion on nasal blood flow in swine. Laryngoscope 109:8-14

53. Viducich RA, Blanda MP, Gerson LW (1995) Posterior epistaxis: clinical features and acute complications. Ann Emerg Med 25(5):592-596

54. Godard JC, Reiter ER (2005) Inpatient management of epistaxis: outcomes and cost. Head Neck Surg 132:707-712 
55. Nikolaou G, Holzman D, Soyka MB (2013) Discomfort and the cost in epistaxis treatment. Eur Arch Otorhinolaryngol 270:2239-2244

56. Brinjikji W, Kallmes DF, Cloft HJ (2013) Trend is epistaxis embolisation in the United States: a study of the Nationwide Inpatient Sample 2003-2010. J Vasc Interv Radiol 24:969-973

57. Elahi MM, Parnes LS, Fox AJ, Pelz DM, Lee DH (1995) Therapeutic embolisation in the treatment of intractable epistaxis. Arch Otolaryngol Head Neck Surg 121:65-69

58. Moreau S, De Rugy MG, Babin E, Courtheoux P, Valdazo A (1998) Superselective embolisation in intractable epistaxis: review of 45 cases. Laryngoscope 108:887-888
59. Leppänen M, Seppänen S, Laranne J, Kuoppala K (1999) Microcatheter embolisation of intractable idiopathic epistaxis. Cardiovasc Interv Radiol 22:499-503

60. Oguni T, Korogi Y, Yasunaga T et al (2000) Superselective embolisation for intractable idiopathic epistaxis. British J Radiol 73:1148-1153

61. Christensen NP, Smith DS, Barnwell SL, Wax MK (2005) Arterial embolisation in the management of posterior epistaxis. Otolaryngol Head Neck Surg 1333:748-753

62. Fukutsuji K, Nishiike S, Aihara T et al (2008) Superselective angiographic embolisation for intractable epistaxis. Acta Otolaryngol 128:556-560 